\title{
A PROBABILISTIC APPROACH FOR THE WAVES GENERATED BY A SUBMARINE LANDSLIDE
}

\author{
Patrick J. Lynett ${ }^{1}$ and Arturo Jimenez Martinez ${ }^{2}$
}

\begin{abstract}
A numerical study aimed at probabilistically assessing the coastal hazard posed by tsunamis induced by onedimensional submarine rigid landslides that experience translational failure is presented. The numerical model here utilized is the finite difference recreation of a linear, fully dispersive mild-slope equation model for wave generation and propagation. This recreated model has the capability to simulate submarine landslides that detach into multiple rigid pieces as failure occurs. An ad-hoc formulation describing the combined space-time coherency of the landslide is presented. Monte Carlo simulations are employed, with an emphasis on the shoreward-traveling waves, to construct probability of exceedance curves for the maximum dimensionless wave height from which wave statistics can be extracted. As inputs to the model, eight dimensionless parameters are specified both deterministically in the form of parameter spaces and probabilistically with normal distributions. Based on a sensitivity analysis, the results of this study indicate that submarine landslides with large width to thickness ratios and coherent failure behavior are most effective in generating tsunamis. Failures modes involving numerous slide pieces that fail in a very compact fashion, however, were observed to induce bigger waves than more coherent landslides. Rapid weakening in tsunami generation potential for some of the parameter combinations suggests that the hazard posed by submarine landslide tsunamis is strongly dependent on source features and local conditions and is only of concern for landslides of substantial dimensions.
\end{abstract}

Keywords: landslide; tsunami; Monte Carlo; mild slope equation

\section{INTRODUCTION}

While not yet widely employed for tsunami impact studies, the Probabilistic Tsunami Hazard Assessment (PTHA) approach appears to be the inevitable future methodology for quantifying tsunami hazard. With PTHA, the user would be provided a probabilistic set of some characteristic quantity, such as runup or momentum flux, which would then be used to determine design loadings or used as the hazard index variable within a risk analysis (Geist and Parsons, 2005). The foundation for PTHA comes from Probabilistic Seismic Hazard Assessment (PSHA), a widely used method that provides, for example, a ground shaking or acceleration that has a $2 \%$ chance of exceedence over 50 years. There is not yet a widely accepted equivalent quantity for tsunami, although there have recently been a handful of studies demonstrating the approach for tsunamis generated by subduction zone earthquakes (e.g. Gonzalez et al., 2009).

A probabilistic assessment of earthquake-generated tsunami hazard is currently feasible due to available seismic information and a "simple" generation mechanism; however this is likely not the case for landslide-generated tsunami hazard. A lack of both geophysical data and fundamental understanding of the generation mechanism make probabilistic input distribution functions and logic trees highly subjective, and arguably so poorly constrained that any result is meaningless. It is the purpose of this study to better describe, in a probabilistic manner, one of the main inputs required of a PTHA. Here we attempt to develop a method to generate wave height exceedence curves for a given landslide by incorporating uncertainties on the slide motion description.

\section{BACKGOUND \& METHODOLOGY}

In light of the dispersive behavior of tsunamis induced by underwater landslides, Tinti et al. (2006) developed a Lagrangian analytical model that incorporates seafloor deformations characteristic of submarine landslides and the frequency filtering effects caused by the water column. In their model, seafloor alterations are incorporated through a forcing function defined as the time derivative of the water depth while filtering of high wave frequencies is implemented by means of a filter function. The purpose of the latter function is to act as a low-pass frequency filter and favor the reproduction of long waves. The absence of the filter function would suggest that bottom disturbances are purely transmitted to the free surface. However, the time-scale of submarine landslides makes it incorrect to assume that the changes in the ocean bottom instantaneously transfer to the free surface, though it is a valid assumption for coseismic tsunamis. The product of the forcing and filtering functions constitutes the

\footnotetext{
${ }^{1}$ University of Southern California, Tsunami Research Center, Los Angeles, CA, USA

${ }^{2}$ Moffat and Nichol, Houston, TX, USA
} 
source term of Tinti et al. (2006) which was utilized to simulate the landslide tsunami that struck the island of Stromboli, Italy on December 30, 2002.

Similarly, but following more closely the discussion of Ward (2001), Kervella et al. (2007) stress upon the importance of incorporating frequency-dispersion capabilities to submarine landslide tsunami models. They support this postulate by comparing 3-D linear and nonlinear tsunami generation models that do and do not account for frequency dispersion. Their approach with respect to the linear models is based on two crucial features of Ward's work: the use of a spectral technique to solve the governing equation and boundary conditions and the utilization of wave number, rather than a seafloor length scale, in the formulation of the filter function. Kervella et al. (2007) concluded their study by stating that NSWE models are not able to reproduce the frequency dispersive behavior of tsunamis, but that linear theory inclusive of dispersive manifestations is more appropriate in this regard. A synopsis of the mentioned studies and their findings suggests that a model for the study of submarine-landslide tsunamis could be formulated using linear theory, but would have to account for frequency dispersion effects in the generation region, grant acceptable accuracy, and be applicable to large domains. To a large extent, these criteria can be satisfied with a model governed by the Mild-Slope Equation (MSE).

Similar to the approach employed to reduce the computational cost of the Navier-Stokes equations, Mild-Slope Equation models offer a simpler alternative to potential flow models. Proposed by Eckart in 1952 and derived by Berkhoff in 1972, the MSE is a depth-integrated version of the Laplace equation operating under the assumption of inviscid flow and mildly-varying bottom slopes. The "mild-slope" acronym surges from assuming that, within a wavelength, the rate of change in depth is small (Demirbilek and Panchang 1998). Regarding their functionality, MSE models offer a mixture of the benefits and limitations of the SWE and Boussinesq models. Most commonly found in their linear form, MSE models are able to simulate small-amplitude wave environments where wave propagation from deep to shallow water and wave scattering effects (i.e., refraction, reflection, and diffraction) are encountered. The main advantages of an MSE model over Boussinesq approximations is that the former covers a wider range of water depths due to its natural incorporation of frequency dispersion and requires less computational effort. In view of these attributes, the MSE has been embraced as a suitable tool for describing coastal wave climate and wave fields in the vicinity of coastal structures and islands (Demirbilek and Panchang 1998). In particular, owed to its dispersion inclusiveness, MSE models are suitable for the simulation of submarine landslide tsunamis.

By incorporating ground motion, Bellotti et al. (2008) developed a linear MSE model capable of reproducing small-amplitude transient waves and frequency dispersion effects caused by changes in the ocean bottom. The forcing function of their model consists of the bottom depth chage term, as proposed by Tinti et al. (2006). Regarding the solution approach to their MSE model, Bellotti et al. (2008) adopt the technique of Kervella et al. (2007) who solve their mathematical problem in the frequency domain by means of a Fourier Transform. To validate their model, Bellotti et al. (2008) recreate the propagation of the December 30, 2002 Stromboli tsunami, obtaining results congruent with the findings of Tinti et al. (2006) and clearly showing the reproduction of frequency dispersion effects when comparing their solutions to those of a long wave model.

Refining the suitability of the linear MSE model of Bellotti et al. (2008) to recreate tsunamis originated by underwater landslides, Cecioni and Bellotti (2010a) incorporated the low-pass filtering influence of the water column into the structure of the source term. Their filter function is the one investigated by Tinti et al. (2006), but with the modification suggested by Kervella et al. (2007) with respect to using wavelength rather than landslide characteristic length. A Fourier Transform is then applied to obtain the elliptic MSE.

Cecioni \& Bellotti (2010a) proceeded to demonstrate the improvements made to the MSE model of Bellotti et al. (2008) by comparing free surface elevations against a 3- D Laplace equation solver. In addition to exposing its computational efficiency, the outcome of this comparison validated the solution accuracy of the MSE model, which turned out to be comparable to that of a Boussinesq equation. At the same time, the satisfactory results were suggestive that the filter function proposed by Kervella et al. (2007) was the appropriate means to recreate the low-pass filtering character of the water column. 
Among their concluding remarks, Cecioni and Bellotti (2010a) motivated the use of the fully dispersive MSE model in early tsunami warning systems.

By numerically recreating the linear MSE model of Cecioni and Bellotti (2010a) using a finitedifference scheme, this research aims at probabilistically analyzing the shoreward-traveling waves generated by 1-D submarine rigid landslides that experience translational failure. Deviating from the failure modes found in the literature, the modeled landslides possess the capability to fail either as a single rigid mass or as a user specified number of rigid pieces. As inputs to the model, eight dimensionless slide parameters influencing tsunami generation will be considered, namely: bottom slope, slide width, slide initial depth, number of pieces in which the slide detaches, slide failure delay, and specific gravity, drag coefficient, and added-mass coefficient of the slide mass. The latter three inputs will be randomly selected using normal distributions while the rest will be deterministically chosen and organized in the form of a parameter space.

To evaluate the free surface response to the various parameter combinations, Monte Carlo simulations will be used to obtain probability of exceedance curves for the maximum dimensionless wave height from which wave statistics will be extracted. In addition, nondimensional relations between wave period and maximum wave height will also be provided. The results of the Monte Carlo simulations will then be presented for a parameter subspace with constant values and a parameter space in which one of the inputs is singularly varied. Tsunami hazard assessment will then advanced by providing insight on the dependency of tsunami generation on the different slide parameters and by providing first-order estimation for nearshore design of the height of the waves that may be triggered by underwater landslides. Landslide triggering, wave breaking and run-up are beyond the capabilities of the recreated model; hence, the present study is limited to wave generation and propagation.

The rest of this manuscript is organized in the following manner. First, recreation and validation of the 1-D and 2-D models are covered along with the equations of motion governing slide dynamics. Next, the Monte Carlo simulations are addressed in terms of their organization, numerical setup, and selection of deterministic and random inputs. The interpretation of the resulting probability distributions is then overseen as well as the inspection of the degree of influence of the different slide parameters on tsunami generation. Finally, conclusions are drawn and future developments suggested.

\section{RESULTS AND DISCUSSION}

First, the MSE model will be compared with previously published Boussinesq and Potential flow results, as found in Lynett and Liu (2002). The profile of the slide is shown in Figure 1. This is a smooth slide that travels downslope following solid body motion. Comparison with various nonlinear and dispersive models are shown in Figure 2. Near the generation area (Fig. 2a), excellent agreement is discerned between the various models at all locations within the domain. At $t=3.0 \mathrm{~s}$ (Fig. 2b), quite good agreement is still observed, though the solution provided by the recreated MSE model slightly undervalues the trough that travels with the slide $(3<\mathrm{x}<4)$. The shoreward propagating wave, however, is well captured. At a later time, Fig. 2c shows a more pronounced underestimation of the trough above the slide by the recreated MSE model with respect to the BIEM and the high-order Boussinesq model. Contrary to the model of Lynett and Liu (2002), the recreated MSE model appears to remain stable as deeper water is entered due to its fully dispersive character. By the time $t=5.86 \mathrm{~s}$ is reached (Fig. 2d), nonlinear effects are more evidently manifesting, which prevents the recreated MSE model from offering a precise match. Nonetheless, the recreated MSE model again demonstrates its full inclusion of dispersion effects by adequately propagating the slide driven trough into deeper water.

The results shown in Fig. 2 are suggestive that in the generation region, nonlinear effects are of secondary importance and that the recreated MSE model captures the free surface response in the initial failure stages very well. These nonlinear effects, however, do seem to acquire relevance as the slide continues its trajectory down slope. In addition, Fig. 2 corroborated the adequacy of the recreated MSE model to simulate wave propagation into deep water. Therefore, given the exemplary agreement in the initial failure stages (Fig. 3a and 3b) and the good reproduction of dispersion effects throughout the free 
surface evolution, the results shown in Fig. 3 are considered satisfactory and affirm the validity of the 1D recreated MSE model.

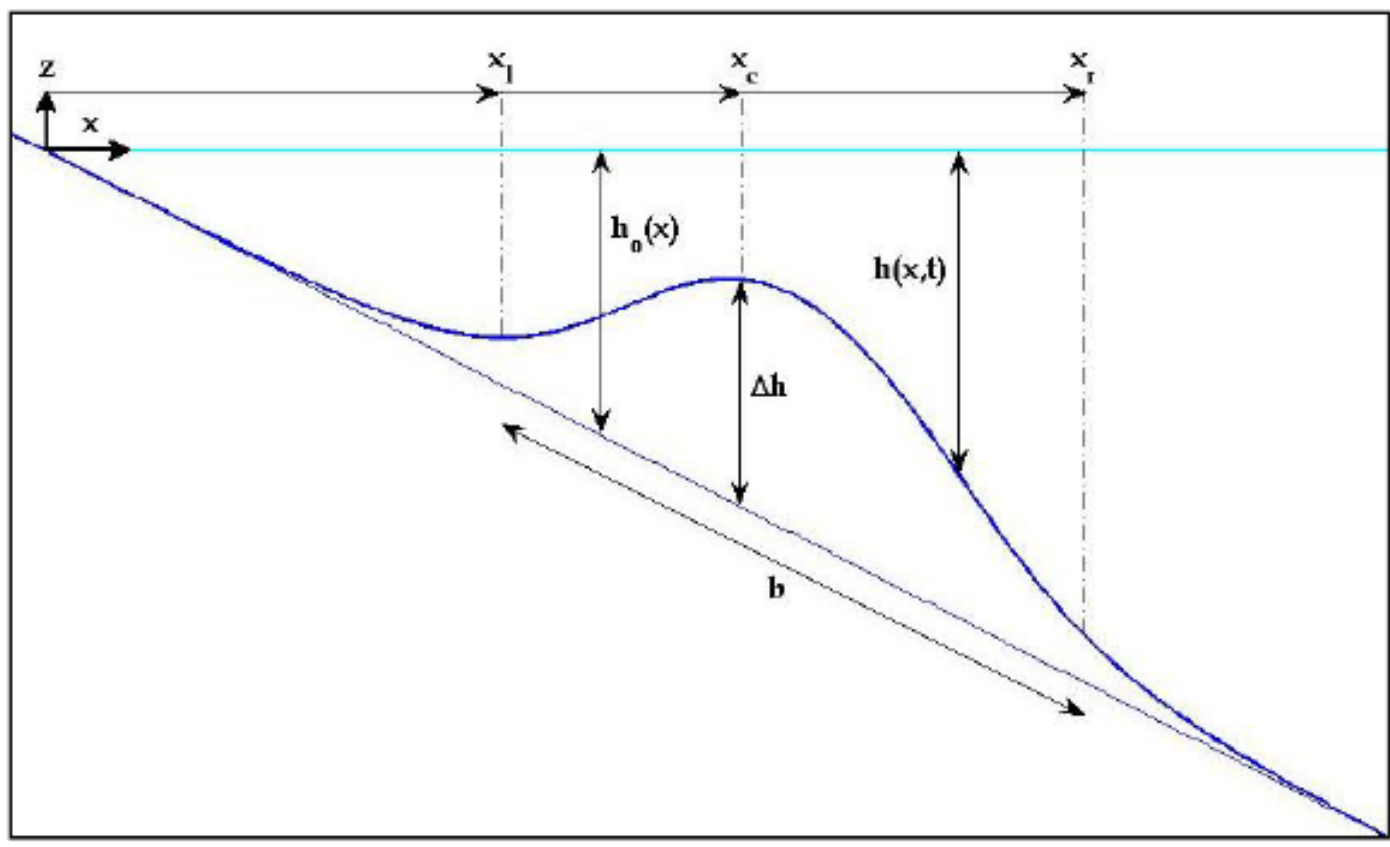

Figure 1. Submarine Landslide Set-Up for Comparison of 1-D MSE Model against Various Nonlinear Models
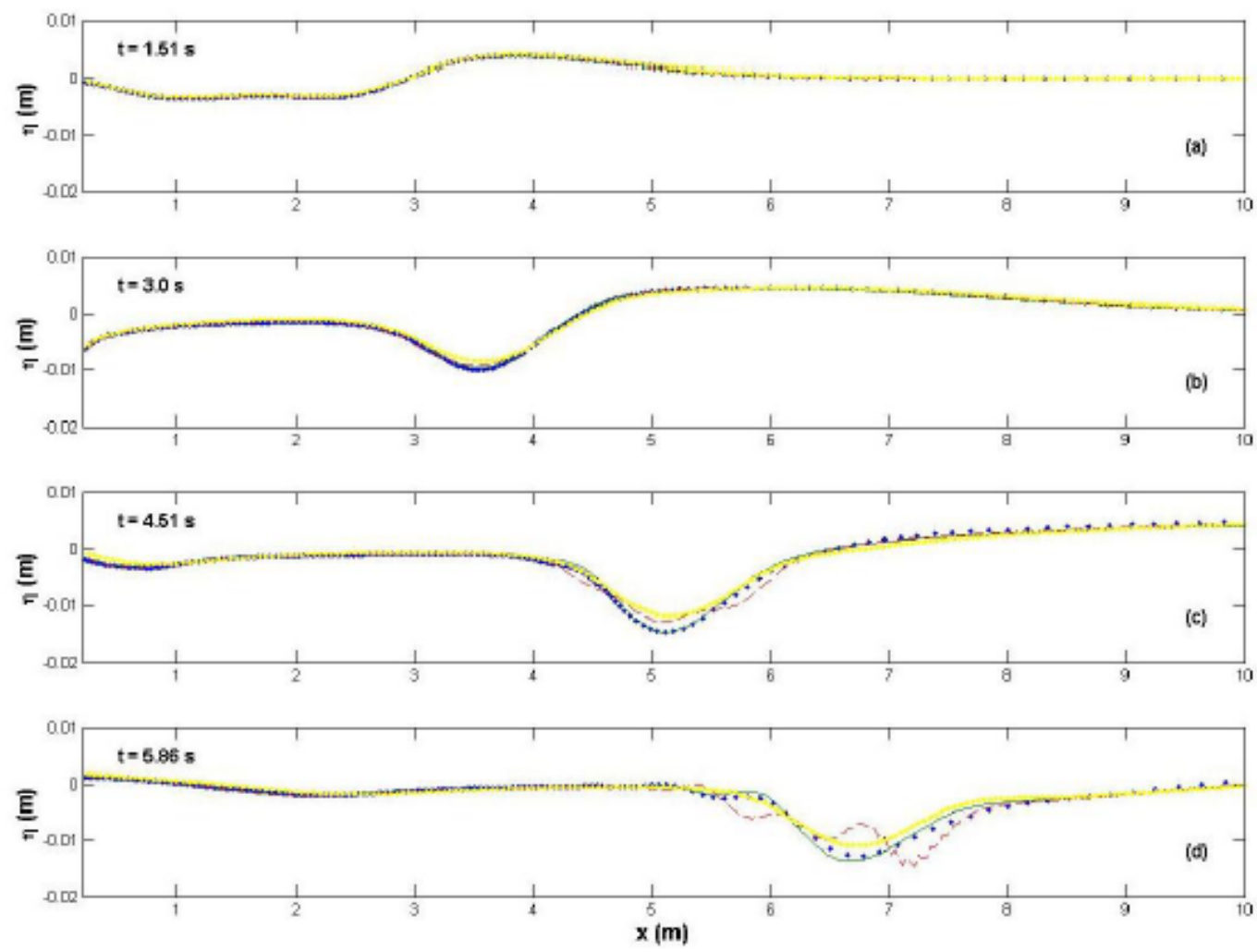

Figure 2. Comparison between the Nonlinear, Weakly Dispersive Depth-Integrated Model (Dashed Line) of Lynett and Liu (2002), the High-Order Boussinesq Model (Solid Line) of Fuhrman and Madsen (2009), the BIEM (Dots) as Described in Lynett and Liu (2002), and the Recreated MSE Model (Dotted Solid Line). 
Now, we attempt to use to model to assess the statistical variability of submarine generated wave heights due to variations in the slide motion. As inputs to the model, eight dimensionless parameters are specified both deterministically in the form of parameter spaces and probabilistically with normal distributions. Figure 3 provides the basic setup and result of the approach. In the top subplot, we see that a single "specified" landslide is allowed to break up into three different slide pieces; Nc=3 for this example.

- Deterministic Inputs:

- $\quad N c$ - Number of detached pieces ( $\mathrm{Nc}_{\mathrm{c}}=3$ in figure)

- $\quad b$ - slide width along slope to thickness ratio

- doI - depth above slide center to thickness ratio

- $\quad$ slope - bottom slope

- delay-dimensionless factor scaling failure duration
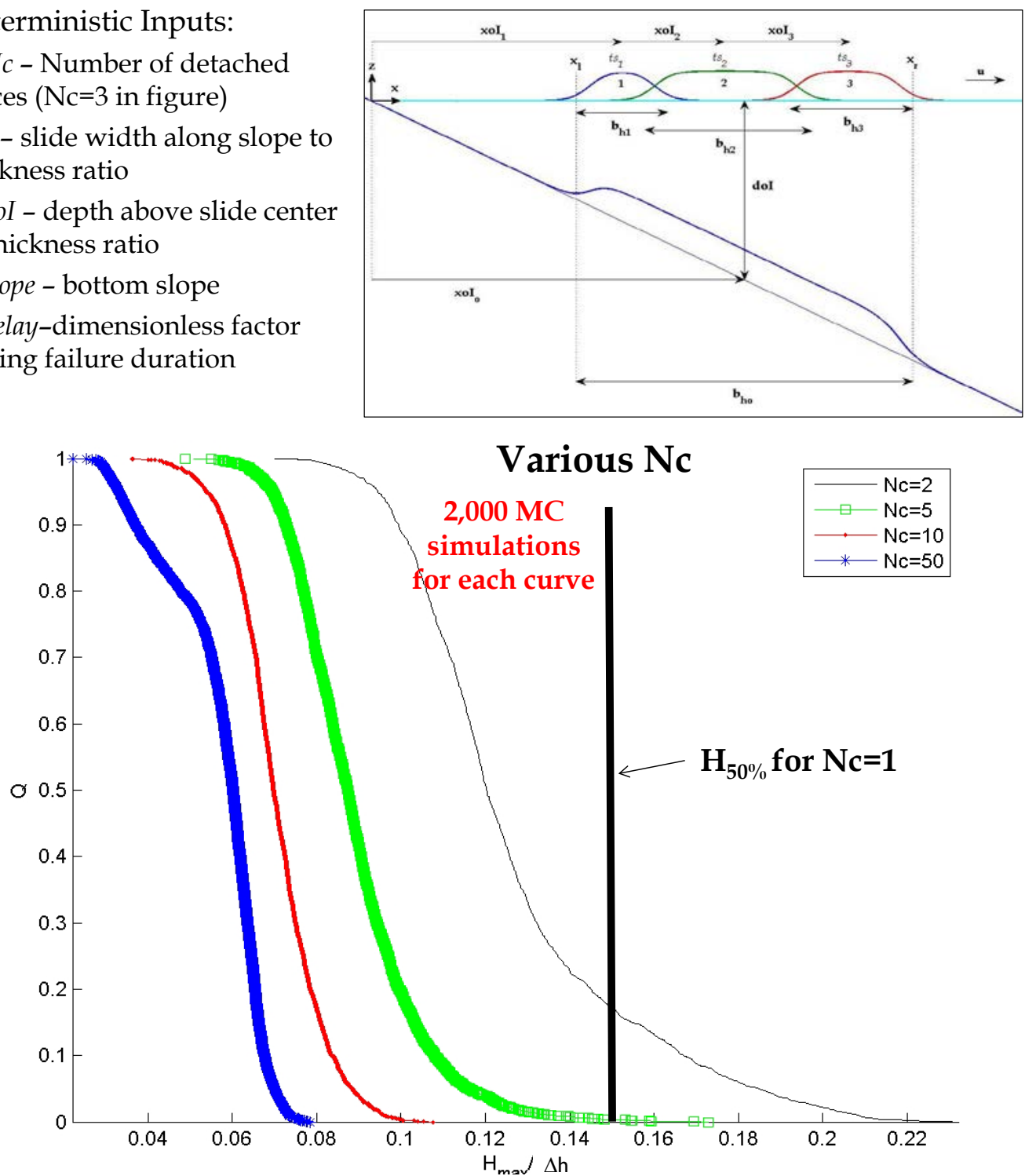

Figure 2. Setup and example results for wave height generation by landslide assessment. In the lower plot are probability of exceedence $(\mathrm{Q})$ curves for different Nc values, with the horizontal axis showing the generated wave height (Hmax) scaled by the initial slide thickness (

Immediately obvious is that slide masses that break into fewer pieces have the potential, on average, to generate larger wave heights. On the other hand, for certain unlikely scenarios, it is possible for a slide that disintegrates into 2 or 3 pieces to in fact generate a larger landward traveling wave than the single coherent slide. The reason for this is that the waves generated by the individually moving pieces can superimpose if the timing is "right," generating a concentration of wave energy larger than that possible 
with the single slide. Such events, albeit unlikely, are very important for PTHA type studies, particularly when the facility of interest requires very low risk and recurrence, such as a Nuclear Power Plant.

\section{CONCLUSIONS}

The presented model has the capability of simulating submarine landslides that detach into multiple rigid pieces as failure occurs. Monte Carlo simulations are employed, with an emphasis on the shoreward-traveling waves, to construct probability of exceedance curves for the maximum dimensionless wave height, from which wave statistics can be extracted. As inputs to the model, eight dimensionless parameters are specified both deterministically in the form of parameter spaces and probabilistically with normal distributions. In agreement with the general conception found in the literature, the results of the Monte Carlo simulations are suggestive that tsunamis generated by submarine landslides are strongly a function of the attributes of the source, the ocean bottom, and the dynamics of the moving mass. Attempting to recognize the extent at which these failure aspects control tsunami generation, a sensitivity analysis for the deterministic parameters was conducted. The presented results also provided valuable insight on the overall hazard posed by submarine landslides. Previous probabilistic studies have labeled this hazard as low, in large part due to their focus on specific geographical regions. The results of this study are representative of a hazard of rather low character, but that can significantly escalate its potential when a favorable combination of physical attributes and failure compactness of the submarine landslide occur. Recall, also, that the presented hazard assessment does not account for the nonlinear processes or wave focusing effects that will certainly act on the tsunami as it reaches the coast. Nevertheless, this study does provide new insight on the general perception submarine landslide tsunamis and their unquestionable threat to the coast. For additional and complete information, the reader is directed to the $2^{\text {nd }}$ authors MS thesis, included online as Supplementary Material.

\section{ACKNOWLEDGMENTS}

This effort was partially supported by Grants from the USGS and NRC.

\section{REFERENCES}

Assier Rzadkiewicz, S., Mariotti, C., Heinrich, P. (1997). "Numerical simulation of submarine landslides and their hydraulic effect.” J. Wtrwy., Port, Coast, and Oc. Engrg., ASCE, 123(4), 149 157.

Bellotti, G., Cecioni, C., and De Girolamo, P. (2008). "Simulation of small- amplitude frequency-dispersive transient waves by means of the mild-slope equation.” Coastal Engrg. 55, 447 $-458$.

Cecioni, C. and Bellotti, G. (2010a). "Modeling tsunamis generated by submerged landslides using depth integrated equations.” Appl. Ocean Res., 32(3), 343 - 350.

Cecioni, C. and Bellotti, G. (2010b). "Inclusion of landslide tsunamis generation into a depth integrated wave model.” Nat. Hazards Earth Syst. Sci., 10, 2259 - 2268.

Fuhrman, D.R. and Madsen, P.A. (2009). "Tsunami generation, propagation, andrun-up with a highorder Boussinesq model.” Coastal Engrg., 56, 747 - 758.

Lynett, P.J. and Liu, P.L.F. (2002). “A numerical study of submarine-landslide- generated waves and run-up.” P. Roy. Soc. London, 458, 2885 - 2910.

Lynett, P.J. and Liu, P.L.F. (2005). "A numerical study of the run-up generated by three-dimensional landslides.” J. Geophys. Res., 110(C03006), doi:10.1029/2004JC002443.

Tinti, S., Pagnoni, G., and Zaniboni, F. (2006). "The landslides and tsunamis of the30th of December 2002 in Stromboli analyzed through numerical simulations.”Bulletin of Volcanology 68 (5), 462 479.

Ward, (2001). S.N. “Landside tsunami”. J. Geophys. Res., 106(6),11,201 - 11,215. Watts, P. (1998). "Wavemaker curves for tsunamis generated by underwaterlandslides.” J. Wtrwy., Port, Coast, and Oc. Engrg., ASCE, 124(3), 127 - 137. 